\title{
Controlled loading and release of beta-lactoglobulin in calcium-polygalacturonate hydrogels
}

Aline Maire du Poset ${ }^{a, b, c}$, Mikaela Börjesson ${ }^{b, d}$, Céline Rameau ${ }^{a}$, Claire MadeleinePerdrillat $^{b}$, Adrien Lerbret ${ }^{a}$, Camille Loupiac ${ }^{a, b}$, Fabrice Cousin ${ }^{b^{*}}$, Ali Assifaoui ${ }^{*}$

a Univ. Bourgogne Franche-Comté, AgroSup Dijon, PAM UMR A 02.102, F-21000 Dijon, France

b Laboratoire Léon Brillouin, Université Paris-Saclay, CEA-Saclay, 91191 Gif-sur-Yvette, France

c Synchrotron SOLEIL, L’Orme des Merisiers, BP48 St Aubin, 91192 Gif-sur-Yvette, France

${ }^{\mathrm{d}}$ Chalmers Univ. of Tech., Department of Chemistry and Chemical Engineering, Kemivägen 10, SE-412 96 Göteborg, Sweden 


\section{SANS measurements of BLG-PolyGalA aqueous mixtures prior to gelation}

Figure S1 shows the same SANS data as those of Figure 2 in main text for BLG $(10 \mathrm{~g} / \mathrm{L})$ and mixtures P10B10, P20B10 and P20B16. In order to compare the scattered intensity of the mixtures to that of the free BLG, IBLG, the scattered intensity for the mixtures were multiplied by the concentration factors (1, 1 and 1.6 for P10B 10, P20B10 and P20B16, respectively). The results reveal a perfect overlap between the normalized Imixture and IBLG.

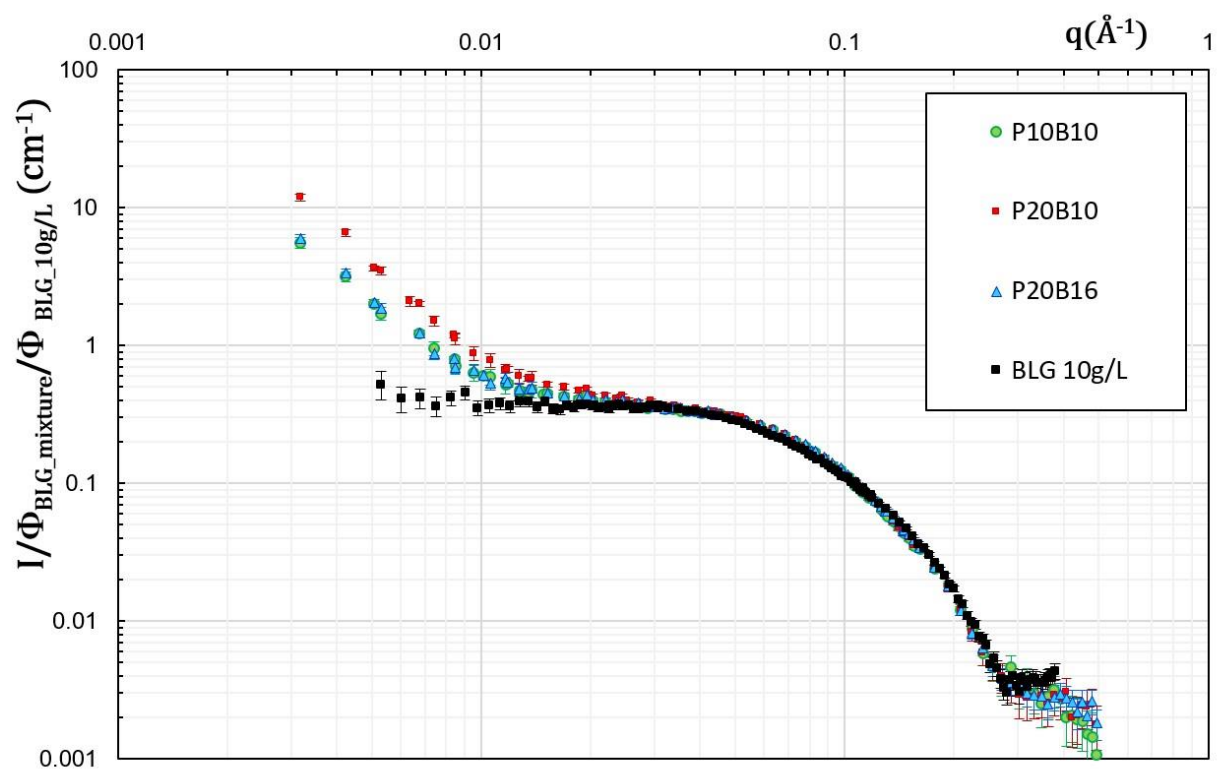

Figure S1. SANS spectra of the P10B10, P20B10, and P20B16 mixtures normalized by the BLG concentration ratio between mixtures and the pure BLG solution $(10 \mathrm{~g} / \mathrm{L})$. The spectra of the pure BLG solution $(10 \mathrm{~g} / \mathrm{L})$ is also shown for comparison. 


\section{SANS measurements of BLG-PolyGalA hydrogels}

Figure S2 shows the same SANS data as those of Figure 3 in main text for BLG $(10 \mathrm{~g} / \mathrm{L})$ and Ca-P10B10, Ca-P20B10 and Ca-P20B16 hydrogels. In order to compare the scattered intensity of the hydrogels to that of the free BLG, the scattered intensity for the hydrogels were divided by constants so that all data perfectly overlap in the large q region. The constants allowed to determine the real concentration of BLG in hydrogels presented in table 1 of main text.
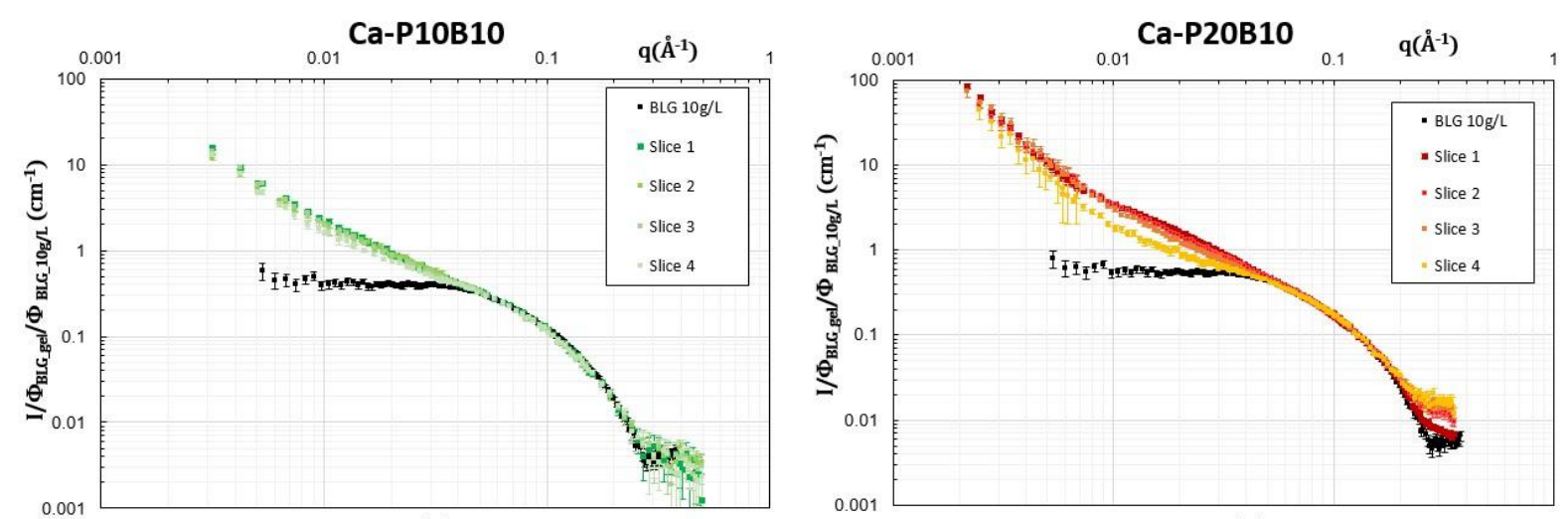

(a)

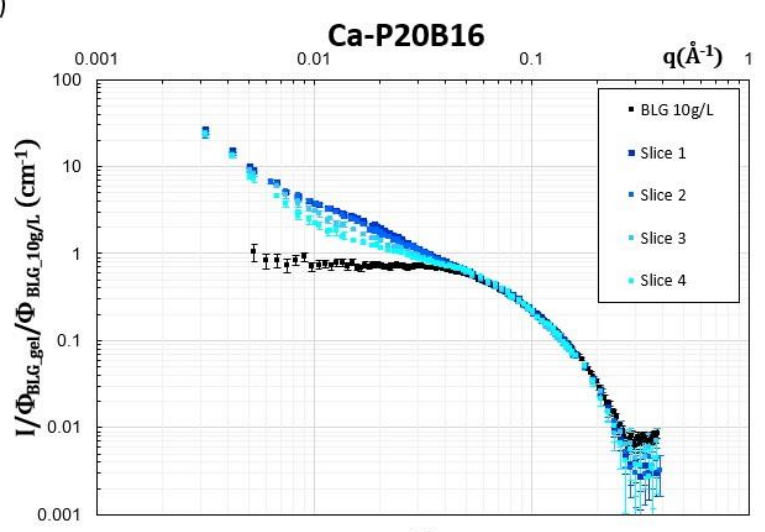

(b)

(c)

Figure S2. SANS spectra of the different slices of mixed hydrogels normalized by the BLG concentration ratio between slices and the pure BLG solution (10 g/L). (a) Ca-P10B10; (b) Ca-P20B10; (c) Ca-P20B16. The spectrum of the pure BLG solution $(10 \mathrm{~g} / \mathrm{L})$ is also shown for comparison and has been scaled in (c) to take into account the higher BLG concentration of mixtures.

Figure S3 compares the SANS data and modelling of Ca-P20B10 and Ca-P20B16 hydrogels to those of a reference Ca-P20 sample for every slice of the hydrogels. 

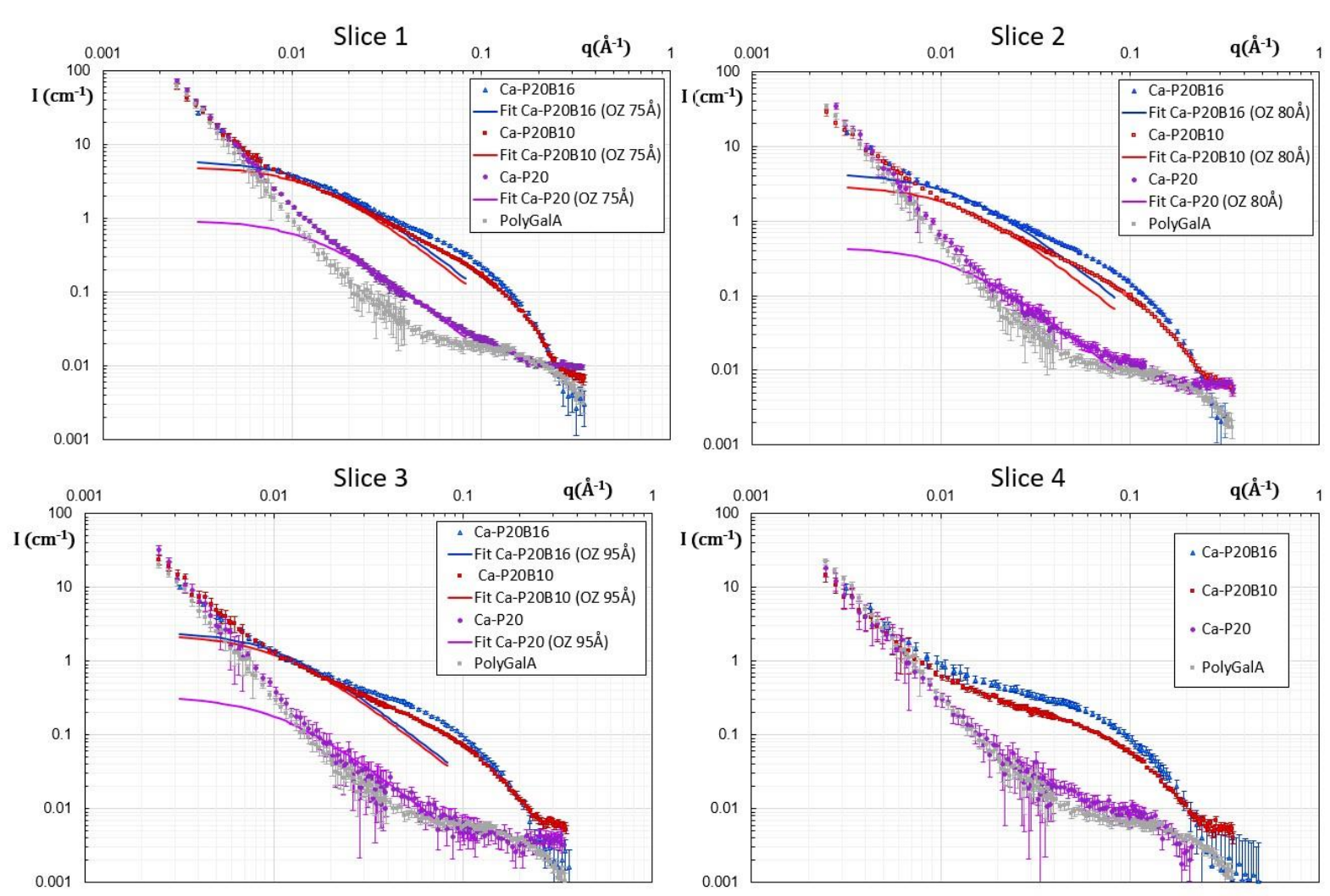

Figure S3. Comparison of the SANS spectra and fitting by a Lorentzian function (Ornstein-Zernike), slice by slice, of the Ca-P20B10, Ca-P20B16 and control Ca-P20 samples. Data are compared to the spectra of pure polyGalA $(20 \mathrm{~g} / \mathrm{L})$ that have been multiplied to a constant factor corresponding to the ratio of concentration between the control Ca-P20 sample and the solution for every panel. 


\section{Mechanical properties of the pure and mixed hydrogels}

Figure S4 shows the Young modulus of the Ca-P10B10 hydrogel as a function of the thickness. Data are compared with those for the pure polyGalA hydrogel (Ca-P20) and for mixed hydrogels (Ca-P20B10 and Ca-P20B16) already presented in main text. The Young modulus is influenced only by the polyGalA concentration and not by the presence of BLG in the mixed hydrogels.

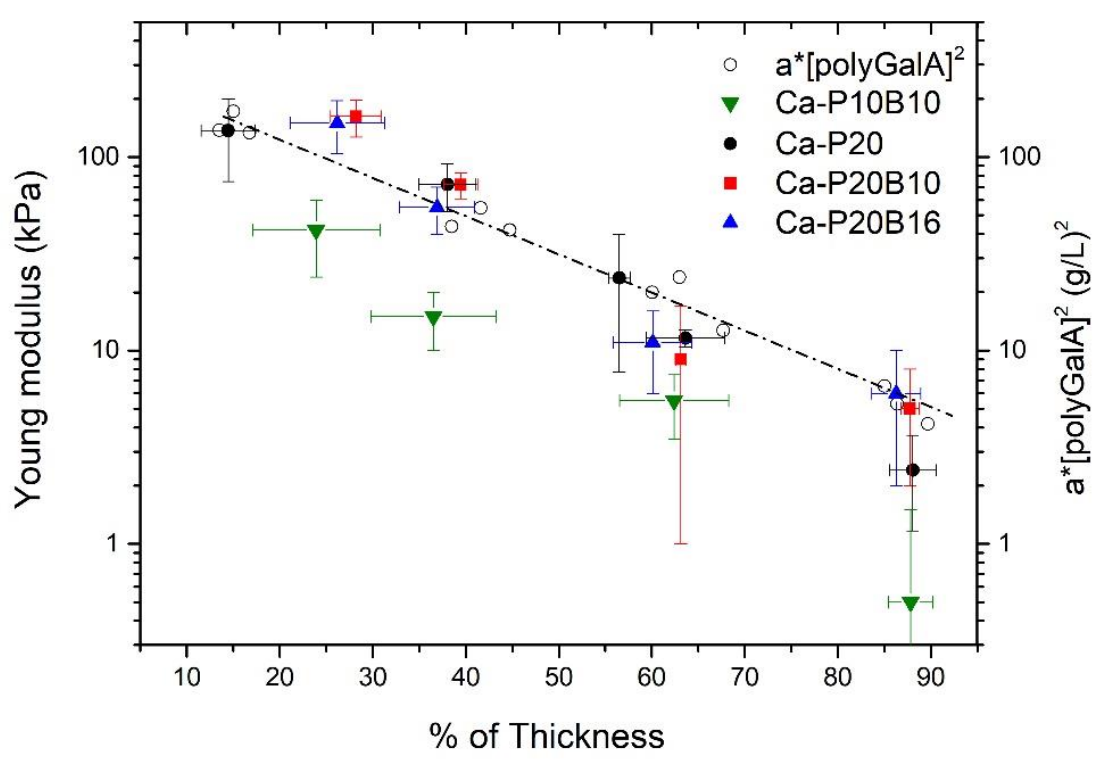

Figure S4. Evolution of the Young modulus (E) as a function of the slice distance to the membrane along the hydrogels (expressed in \% of thickness) for the pure polyGalA hydrogel (Ca-P20) and for mixed hydrogels (Ca-P10B10, Ca-P20B10 and Ca-P20B16). Empty circles represent the evolution of the square of the polyGalA concentration in the Ca-P20 hydrogel (times a constant a equal to 0.105 ) as function of the gel thickness. The black dash line shows the relationship $\mathrm{E} \sim 0.105 \mathrm{x}[\text { polyGalA }]^{2}$. 